\title{
Silicon optical modulators for high data rate applications
}

D. Thomson 1', F. Y. Gardes ${ }^{1}$, G. T. Reed ${ }^{1}$, G. Rasigade 2 , M. Ziebell ${ }^{2}$, D. Marris-Morini', L. Vivien², A. Brimont ${ }^{3}$, P. Sanchis ${ }^{3}$, J-M. Fédéli ${ }^{4}$, L. O'Faolain ${ }^{5}$, T. F. Krauss ${ }^{5}$, L. Lever ${ }^{6}$, Z. Ikonic ${ }^{6}$ and R. W. Kelsall ${ }^{6}$.

1- Advanced Technology Institute, University of Surrey, Guildford, Surrey, UK. 2 - Institut d'Electronique Fondamentale, Université Paris-sud XI, ORSAY, France. 3 - Nanophotonics Technology Center, Universidad Politécnica de Valencia, Valencia, Spain. 4 - CEA-LETI, Minatec, CEA-Grenoble, Grenoble, France.

5- School of Physics \& Astronomy, University of St Andrews, North Haugh, St Andrews, UK. 6- Institute of Microwaves and Photonics, University of Leeds, Leeds, UK.

\begin{abstract}
In this work we describe the carrier depletion MZI modulators, slow wave structures for modulation enhancement and the QCSE modulator which are under development in the European HELIOS project and the UK Silicon Photonics project.
\end{abstract}

\section{Introduction}

High performance silicon optical modulators are key to many silicon based photonic applications. Over the previous decade the development seen in the performance of silicon optical modulators has been vast. Several routes to modulation have been used to overcome the lack of a strong electro optic effect in silicon. These include the plasma dispersion effect, III-V hybrid device fabrication, SiGe devices, Polymer and Strain induced electro-optic effects. Reported performances now regularly range from 10Gbit/s up to 40Gbit/s. HELIOS, which is a European FP7 funded project and the UK silicon photonics project (UKSP), funded by the EPSRC both involve the development of the different photonic components required to form photonic circuits with a range of functionality. Within both projects there is strong modulator activity with carrier depletion based modulation, QCSE modulation and structures to gain enhancement of the modulation effect under development.

\section{Carrier depletion modulation}

Optical modulators based upon free carrier depletion are widely regarded as being the simplest approach to achieve high performance modulation in silicon. They operate by reverse biasing a diode structure which is incorporated in or around an optical waveguide. The depletion of free carriers therefore interacts with the propagating light causing a change in phase through the plasma dispersion effect. Within the HELIOS project two phase modulators based upon this approach are under investigation using both PN and PIPIN diodes. Cross sectional diagrams of these devices are shown in figure 1.
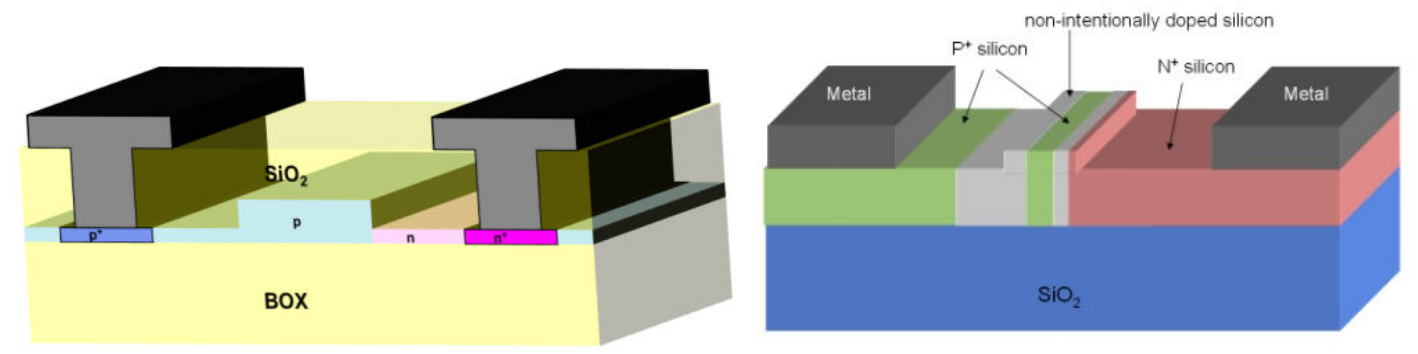

Figure 1 - Modulator in 220nm SOI (left) and in 400nm SOI (right)

The first structure is based in silicon-on-insulator (SOI) of $220 \mathrm{~nm}$ thickness. The waveguide section and the slab to one side is doped $p$ type. The slab on the other side of the waveguide is then doped $n$ type setting up a pn junction at the edge of the waveguide rib. The concentration of the $n$ type doping is made larger than the $p$ type doping such that the depletion region extends mainly into the waveguide during reverse bias. These lightly doped $\mathrm{p}$ and $\mathrm{n}$ type regions extend out to meet highly doped regions which in turn provide ohmic contacts to coplanar waveguide electrodes which are used to drive the device. The device 
has so far demonstrated modulation at 10Gbit/s [1] and more recently 40Gbit/s has been achieved. The second structure has a $p$ type doped slit through the centre of a $400 \mathrm{~nm}$ high rib waveguide which is depleted upon reverse biasing the device. This design gives optimal overlap of the core of optimal mode with the region of varying refractive index thus providing a large modulation efficency with low optical loss. The slab regions to the side of rib are doped $\mathrm{p}$ type on one side and $\mathrm{n}$ type on the other and again contact to coplanar waveguide electrodes to drive the device. 10Gbit/s modulation has been achieved with an $8.1 \mathrm{~dB}$ extinction ratio and $6 \mathrm{~dB}$ of optical loss [2]. A further carrier depletion phase modulator is also under development within the UKSP program. It is also based upon a PN diode and has demonstrated $40 \mathrm{Gbit} / \mathrm{s}$ and $10 \mathrm{Gbit} / \mathrm{s}$ modulation with extinction ratios up to $6 \mathrm{~dB}$ and $7 \mathrm{~dB}$ respectively for both TE and TM polarisations [3].

\section{Structures for modulation enhancement}

Whilst high speed modulation can be achieved in carrier depletion based optical modulators, their efficiency is relatively low and therefore device lengths in the millimetre regime are typically required. In order to minimise the footprint of the devices both ring resonator and photonic crystal structures are under investigation within the projects. The slowlight effect in photonic crystal $(\mathrm{PhC})$ waveguides can dramatically enhance the phase efficiency of carrier based devices. This allows a reduction in the length of a $\mathrm{PhC}$ modulator by the slowdown factor. For example values of 10 have been shown over bandwidths of $10 \mathrm{~nm}$ [4] and a group index of 13.5 over a $14 \mathrm{~nm}$ range [5]. Importantly, by virtue of this reduced length, fewer carriers are required to achieve a given phase change, giving very significant power savings.
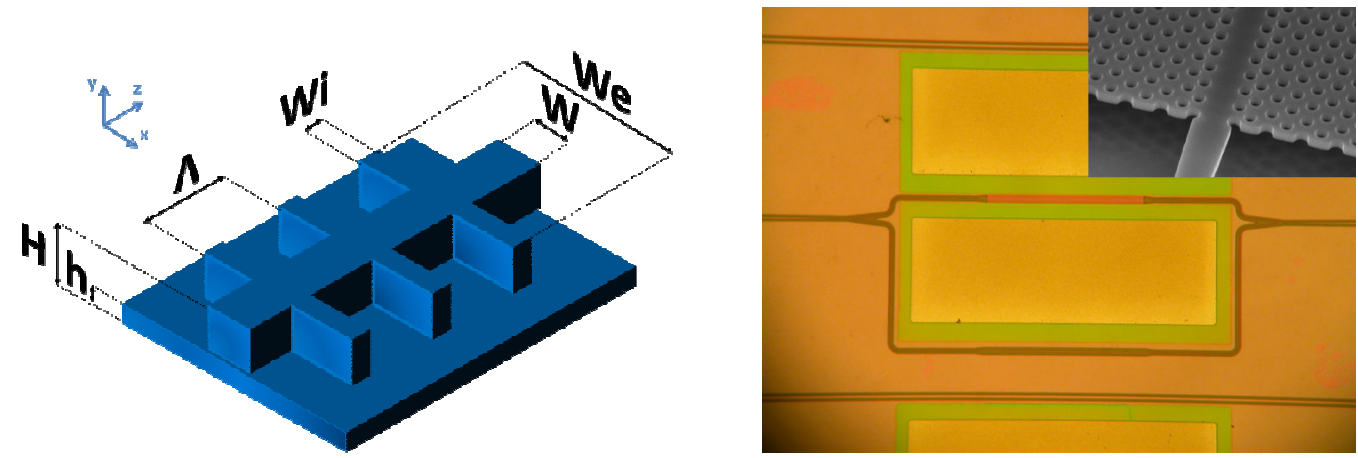

Figure 2 - Diagram of corrugated waveguide sturcture (left), Optical image of an asymmetric PhC MZI with an SEM image of the device shown in the inset (right).

Within the HELIOS project corrugated waveguide structures as shown in figure 2 (left) are under development with the aim of incorporating the carrier depletion modulator shown in the left of figure 1 into a corrugated waveguide. Within the UK Silicon photonics project a more traditional photonic crystal structure as shown in the right hand image of figure 2 is being investigated. Recently an integrated a PIN diode with a PhC modulator achieving a 4 times improvement in electro-optic modulation has been demonstrated, which suggests that very high speed performance can be expected from this device when driven by an RF signal. Within the HELIOS project various types of ring based structures are also under development with the aim of incorporating the carrier depletion based modulator in the right of figure 1 .

\section{QCSE modulation}

The quantum-confined Stark effect (QCSE) describes a shift in the absorption edge of a quantum well heterostructure in response to an electric field applied perpendicular to the plane of the quantum wells. It was first observed in Ge/SiGe multiple quantum well (MQW) systems in 2005 [6], and has since been observed a variety of well dimensions and material compositions (see, e.g., [7]). In order to exploit this phenomenon in a waveguide-integrated device, one of the important concerns is to minimise the insertion loss. Two factors that limit the insertion loss are the sub-band-gap absorption of the Ge/SiGe MQW system, and the coupling between the device and the incoming and outgoing waveguides. Within the UKSP program a model has been developed to determine the absorption spectra in Ge/SiGe MQW 
systems, whereby indirect optical absorption is accounted for either by assuming a bulk matrix element for the $\Gamma \rightarrow L$ scattering rate [8], or by calculating the rate from a virtual $\Gamma-$ valley subband into all available $L$-valley subbands. Using both methods we find that the simulated indirect absorption is significantly less than the measured sub-band-gap absorption found in the literature. Ge/SiGe MQW structures grown on virtual substrates have large threading dislocation densities (TDDs) [9]. It is known that TDDs enhance the sub-band-gap absorption [10], and so it is suggested that there is scope to reduce the subband-gap absorption by further development of SiGe virtual substrate technology. Although some progress has been made towards integration with SOI waveguides [11], waveguideintegrated devices with acceptable performance metrics have not been demonstrated. One issue affecting optical propagation is the mode mismatch between the incoming waveguide and the device. State-of-the-art SOI photonics platforms use thin (400-nm-thick or less) Si waveguide layers; virtual substrates for Ge/SiGe MQW heterostructures are typically 500nm-thick [6,7,9], which, in addition to the height MQW device, means that there is a significant difference in height between the waveguides and the device. We use an eigenmode expansion method to simulate the optical propagation in the modulator, and find that the incoming mode is able to excite multiple modes within the thicker device section. The resulting coherent re-imaging means that it is important to correctly choose to length of the device in order to efficiently out-couple the radiation and hence minimise the insertion loss.

\section{Acknowledgements}

The research leading to these results has received funding from the European Community's Seventh Framework Programme (FP7/2007-2013) under grant agreement $n^{\circ} 224312$ HELIOS and from the EPSRC in the UK to support the UK Silicon Photonics project.

\section{References}

[1] - D. J. Thomson, F. Y. Gardes, G. T. Reed, F. Milesi and J-M Fedeli "High speed silicon optical modulator with self aligned fabrication process," Opt. Express, 18(18), 19064-19069, (2010).

[2] - G. Rasigade, M. Ziebell, D. Marris-Morini, J-M. Fédéli, F. Milesi, P. Grosse, D. Bouville, E. Cassan and L. Vivien, "High extinction ratio $10 \mathrm{Gbit} / \mathrm{s}$ silicon optical modulator," Opt. Express, 19(7), 5827-5832, (2011).

[3] - F. Y. Gardes, D. J. Thomson, G. T. Reed, " $40 \mathrm{~Gb} / \mathrm{s}$ high-speed silicon modulator for TE and TM polarisation," Proceedings of Photonics west 2011, (2011).

[4] - L. O'Faolain, D. M. Beggs, T. P. White, T. Kampfrath, K. Kuipers and T. F. Krauss, "Compact Optical Switches and Modulators Based on Dispersion Engineered Photonic Crystals," IEEE Photonics Journal, 2(3), 404-414, (2010).

[5] - A. Brimont, J. V. Galán, J. M. Escalante, J. Martí and P. Sanchis, "Group-index engineering in silicon corrugated waveguides," Optics letters, 35 (16), (2010).

[6] -Y.-H. Kuo, Y. K. Lee, Y. Ge, S. Ren, J. E. Roth, T. I. Kamins, D. A. B. Miller and J. S. Harris, "Strong quantum-confined Stark effect in germanium quantum-well structures on silicon," Nature, 437, 1334-1336, (2005).

[7] - R. K. Schaevitz, J. E. Roth, S. Ren, O. Fidaner, and D. A. Miller, "Material properties of $\mathrm{Si}-\mathrm{Ge} / \mathrm{Ge}$ quantum wells," IEEE Journal of selected topics in quantum electronics, 14, 10821089, (2008).

[8] - L. Lever, Z. Ikonic, A. Valavanis, J. Cooper and R. Kelsall, "Design of Ge-SiGe quantum confined stark effect electroabsorption heterostructures for CMOS compatible photonics," Lightwave Technology, Journal of, 28(22), 3273 -3281, (2010).

[9] - J. S Harrier and Y.-H. Kuo, "Thin buffer layer for SiGe growth on mismatched substrates," US Patent 077 734, (2007).

[10] - E. Peiner, A. Guttzeit and H.-H. Wehmann, "The effect of threading dislocations on optical absorption and electron scattering in strongly mismatched heteroepitaxial IIIV compound semiconductors on silicon," Journal of Physics: Condensed Matter, 14(48), 13195, (2002).

[11] - S. Ren, Y. Rong, T. I. Kamins, J. S. Harris and D. A. B. Miller, "Integration of germanium quantum well structures on a silicon-oninsulator waveguide platform for optical modulator applications," 2010 7th IEEE International Conference on Group IV Photonics, (2010). 